\title{
Transverse properties of parabolic subgroups of Garside groups
}

\author{
YAGO ANTOLÍN \\ LUIS PARIS
}

\begin{abstract}
Let $G$ be a Garside group endowed with the generating set $\mathcal{S}$ of non-trivial simple elements, and let $H$ be a parabolic subgroup of $G$. We determine a transversal $T$ of $H$ in $G$ such that each $\theta \in T$ is of minimal length in its right-coset, $H \theta$, for the word length with respect to $\mathcal{S}$. We show that there exists a regular language $L$ on $\mathcal{S} \cup \mathcal{S}^{-1}$ and a bijection ev : $L \rightarrow T$ satisfying $\lg (U)=\lg _{\mathcal{S}}(\operatorname{ev}(U))$ for all $U \in L$. $>$ From this we deduce that the coset growth series of $H$ in $G$ is rational. Finally, we show that $G$ has fellow projections on $H$ but does not have bounded projections on $H$.
\end{abstract}

20F36, 20F10

\section{Introduction}

Let $S$ be a finite set. A Coxeter matrix over $S$ is defined to be a square matrix $M=\left(m_{s, t}\right)_{s, t \in S}$ indexed by the elements of $S$, with coefficients in $\mathbb{N} \cup\{\infty\}$, such that $m_{s, s}=1$ for all $s \in S$, and $m_{s, t}=m_{t, s} \geq 2$ for all $s, t \in S, s \neq t$. For each $s, t \in S, s \neq t$, and each integer $m \geq 2$, we set $\Pi(s, t, m)=(s t)^{\frac{m}{2}}$ if $m$ is even and $\Pi(s, t, m)=(s t)^{\frac{m-1}{2}} s$ if $m$ is odd. In other words, $\Pi(s, t, m)$ denotes the word $s t s \cdots$ of length $m$. The Artin group associated with the above Coxeter matrix $M$ is the group $A=A_{M}$ defined by the presentation

$$
\left.A=A_{M}=\langle S| \Pi\left(s, t, m_{s, t}\right)=\Pi\left(t, s, m_{s, t}\right) \text { for all } s, t \in S, s \neq t \text { and } m_{s, t} \neq \infty\right\rangle .
$$

The Coxeter group associated with $M$, denoted by $W=W_{M}$, is the quotient of $A$ by the relations $s^{2}=1, s \in S$.

Artin groups were introduced by Tits [24] as extensions of Coxeter groups. There are few known results valid for all Artin groups, and most of the work in the subject concerns particular families of them. The family concerned by the present paper is the one of Artin groups of spherical type, that is, the Artin groups whose associated Coxeter groups are finite. Seminal work on these groups are Brieskorn [2, 3], BrieskornSaito [4] and Deligne [13] in relation with the so-called discriminant varieties. They 
are involved in several subjects such as singularities, Hecke algebras, hyperplane arrangements, mapping class groups, and there is an extensive literature on them. The leading examples of spherical type Artin groups are the braid groups.

Let $G$ be a group and let $M$ be a submonoid of $G$ such that $M \cap M^{-1}=\{1\}$. Then we have two partial orders $\leq_{L}$ and $\leq_{R}$ on $G$ defined by $\alpha \leq_{L} \beta$ if $\alpha^{-1} \beta \in M$, and $\alpha \leq_{R} \beta$ if $\beta \alpha^{-1} \in M$. For each $a \in M$ we set $\operatorname{Div}_{L}(a)=\left\{b \in M \mid b \leq_{L} a\right\}$ and $\operatorname{Div}_{R}(a)=\left\{b \in M \mid b \leq_{R} a\right\}$. An element $a \in M$ is called balanced if $\operatorname{Div}_{L}(a)=\operatorname{Div}_{R}(a)$. In this case we set $\operatorname{Div}(a)=\operatorname{Div}_{L}(a)=\operatorname{Div}_{R}(a)$. On the other hand, we say that $M$ is Noetherian if, for each $a \in M$, there exists an integer $n \geq 1$ such that $a$ cannot be decomposed as a product of more than $n$ non-trivial factors.

Let $G$ be a group, let $M$ be a submonoid of $G$ such that $M \cap M^{-1}=\{1\}$, and let $\Delta$ be a balanced element of $M$. We say that $G$ is a Garside group with Garside structure $(G, M, \Delta)$ if:

(a) $M$ is Noetherian;

(b) $\operatorname{Div}(\Delta)$ is finite, it generates $M$ as a monoid, and it generates $G$ as a group;

(c) $\left(G, \leq_{L}\right)$ is a lattice.

In this case $\Delta$ is called the Garside element and the elements of $\operatorname{Div}(\Delta)$ are called the simple elements of $G$ for the given Garside structure. The lattice operations of $\left(G, \leq_{L}\right)$ are denoted by $\wedge_{L}$ and $\vee_{L}$, where for $\alpha, \beta \in G$,

$$
\alpha \wedge_{L} \beta=\max _{\leq_{L}}\left(\gamma \in G \mid \gamma \leq_{L} \alpha \text { and } \gamma \leq_{L} \beta\right)
$$

and

$$
\alpha \vee_{L} \beta=\min _{\leq_{L}}\left(\gamma \in G \mid \alpha \leq_{L} \gamma \text { and } \beta \leq_{L} \gamma\right)
$$

Remark that if $\alpha, \beta \in M$, then $1 \leq_{L} \alpha$ and $1 \leq_{L} \beta$ and therefore $1 \leq_{L}\left(\alpha \wedge_{L} \beta\right) \leq_{L}$ $\left(\alpha \vee_{L} \beta\right)$ and thus, $\wedge_{L}$ and $\vee_{L}$ restrict to lattice operations of $\left(M, \leq_{L}\right)$. On the other hand, the ordered set $\left(G, \leq_{R}\right)$ is also a lattice and its lattice operations are denoted by $\wedge_{R}$ and $\vee_{R}$ and these operations also restrict to lattice operations of $\left(M, \leq_{R}\right)$.

This definition is due to Dehornoy-Paris [12]. It is inspired by the work of Garside [16], Epstein et al. [14], Charney [6, 7] and others on braid groups and, more generally, on Artin groups of spherical type. It isolates certain combinatorial characteristics of the braid groups and of the spherical type Artin groups that suffice to show certain algebraic properties such as the existence of a solution to the conjugacy problem, or the bi-automaticity. By Brieskorn-Saito [4] and Deligne [13], the Artin groups of spherical type are Garside groups, and a large part of the study of these groups is made 
now in terms of Garside groups. We refer to Dehornoy et al. [11] for a full account on the theory.

Let $A$ be an Artin group associated with a Coxeter matrix $M=\left(m_{s, t}\right)_{s, t \in S}$. The subgroup $A_{X}$ of $A$ generated by a subset $X$ of $S$ is called a (standard) parabolic subgroup of $A$. By van der Lek [21] we know that $A_{X}$ is itself an Artin group, associated with the Coxeter matrix $M_{X}=\left(m_{s, t}\right)_{s, t \in X}$. Parabolic subgroups play a prominent role in the study of Artin groups in general and in that of Artin groups of spherical type in particular. For example, they are one the main ingredients in the definition/construction of the Salvetti complex for Artin groups, which is one of the most important tools in the study of the $K(\pi, 1)$ conjecture for Artin groups (see for example Godelle-Paris [19] and Paris [22]) and in the calculation of the cohomology of these groups (see for example De Concini-Salvetti [8] and Callegaro-Moroni-Salvetti [5]).

The notion of parabolic subgroup was extended to Garside groups by Godelle [17, 18] as follows. Let $(G, M, \Delta)$ be a Garside structure on a Garside group $G$ and let $\delta$ be a balanced element in $M$. Let $G_{\delta}$ (resp. $M_{\delta}$ ) denote the subgroup of $G$ (resp. the submonoid of $M)$ generated by $\operatorname{Div}(\delta)$. Then we say that $\left(G_{\delta}, M_{\delta}, \delta\right)$ is a parabolic substructure if $\operatorname{Div}(\delta)=\operatorname{Div}(\Delta) \cap M_{\delta}$. Note that this condition implies that $\delta \in \operatorname{Div}(\Delta)$. In this case we say that $G_{\delta}$ is a parabolic subgroup of $G$ and that $M_{\delta}$ is a parabolic submonoid of $M$.

In this paper a parabolic subgroup will be always assumed to be non-trivial.

Let $A$ be an Artin group of spherical type, let $S$ be the standard generating set of $A$, and let $X$ be a subset of $S$. Then, by Brieskorn-Saito [4], the subgroup $A_{X}$ of $A$ generated by $X$ is a parabolic subgroup in Godelle's sense, and all parabolic subgroups of $A$ are of this form.

Remark Let $H$ be a parabolic subgroup of a Garside group $G$ with Garside structure $(G, M, \Delta)$. Then there is a unique parabolic substructure $\left(G_{\delta}, M_{\delta}, \delta\right)$ such that $H=G_{\delta}$. Indeed, the balanced element $\delta$ which defines this parabolic substructure is the greatest element of $\operatorname{Div}(\Delta) \cap H$ for the ordering $\leq_{L}$, hence $H$ determines $\delta$. Similarly, if $N$ is a parabolic submonoid of $M$, then there is a unique parabolic substructure $\left(G_{\delta}, M_{\delta}, \delta\right)$ such that $N=M_{\delta}$, where $\delta$ is the greatest element of $\operatorname{Div}(\Delta) \cap N$ for the ordering $\leq_{L}$. So, we can speak of a parabolic subgroup and/or of a parabolic submonoid without necessarily mentioning the balanced element $\delta$ or the triple $\left(G_{\delta}, M_{\delta}, \delta\right)$.

The results of the present paper are new for Artin groups of spherical type and even for braid groups and, as often now in the theory, the whole study is done in terms of Garside groups. 
Let $G$ be a Garside group with Garside structure $(G, M, \Delta)$. As often in the theory the generating set of $G$ that will be considered in the present paper is the set $\mathcal{S}=\operatorname{Div}(\Delta) \backslash$ $\{1\}$ of non-trivial simple elements. For each $\alpha \in G$ we denote by $\lg (\alpha)=\lg _{\mathcal{S}}(\alpha)$ the word length of $\alpha$ with respect to $\mathcal{S}$. Let $H$ be a parabolic subgroup of $G$. Recall that a right-coset of $H$ in $G$ is a subset of $G$ of the form $H \alpha=\{\beta \alpha \mid \beta \in H\}$, with $\alpha \in G$. Recall also that a (right) transversal of $H$ in $G$ is a subset $T$ of $G$ such that $C \cap T$ is a singleton for every right-coset $C$ of $H$ in $G$. On the other hand, we define the length of a right-coset $C$ of $H$ in $G$ to be $\lg (C)=\min \{\lg (\beta) \mid \beta \in C\}$. In a first step we will determine an explicit transversal $T$ of $H$ in $G$ which satisfies $\lg (\theta)=\lg (H \theta)$ for all $\theta \in T$ (see Theorem 3.2 and Theorem 3.3).

We denote by ev $:\left(\mathcal{S} \cup \mathcal{S}^{-1}\right)^{*} \rightarrow G$ the map which sends each word $U$ to the element of $G$ that it represents. In a second step we will determine a regular language $L$ over $\left(\mathcal{S} \cup \mathcal{S}^{-1}\right)^{*}$ satisfying $\operatorname{ev}(L)=T$, the restriction ev $: L \rightarrow T$ is a bijective correspondence, and $\lg (U)=\lg (\operatorname{ev}(U))$ for all $U \in L$ (see Theorem 4.2). The notion of regular language will be recalled in Section 4.

For each $n \in \mathbb{N}$ we denote by $e(n)=e_{G, H, \mathcal{S}}(n)$ the number of right-cosets of $H$ in $G$ of length $n$. The coset growth series of $H$ in $G$ is defined to be the formal series $\operatorname{Gr}_{G, H, \mathcal{S}}(t)=\sum_{n=0}^{\infty} e(n) t^{n}$. By the above $e(n)$ is the number of words of length $n$ in the regular language $L$. So, $\mathrm{Gr}_{G, H, \mathcal{S}}(t)$ is equal to the growth series of $L$. As we know that the growth series of a regular language is rational (see Flajolet-Sedgewick [15] for example), the formal series $\operatorname{Gr}_{G, H, \mathcal{S}}(t)$ is rational.

The starting point of this work was the paper [1] where the first author studies the triples $(G, \mathcal{S}, H)$ where $G$ is a group, $\mathcal{S}$ a finite generating set of $G$ and $H$ is a subgroup of $G$ such that $(G, \mathcal{S})$ has the falsification by fellow traveller property and has fellow projections and/or bounded projections on $H$. These notions will be recalled in Section 5. In particular, it is proved in Antolín [1] that, if $(G, \mathcal{S})$ has the falsification by fellow traveller property and has fellow projections on $H$, then the language $\operatorname{Geo}(H \backslash G, \mathcal{S})=$ $\left\{U \in\left(\mathcal{S} \cup \mathcal{S}^{-1}\right)^{*} \mid \lg (U)=\lg (H \operatorname{ev}(U))\right\}$ is regular. It is also proved in Antolín [1] that, if $(G, \mathcal{S})$ has the falsification by fellow traveller property and has bounded projections on $H$, then the coset growth series $\mathrm{Gr}_{G, H, \mathcal{S}}(t)$ is rational. Let $G$ be a Garside group, $\mathcal{S}=\operatorname{Div}(\Delta) \backslash\{1\}$ and let $H$ be a parabolic subgroup of $G$. We know by Holt [20] that $(G, \mathcal{S})$ has the falsification by fellow traveller property. So, it is natural to ask whether $G$ has fellow projections and/or bounded projections on $H$.

In the present paper we show that $(G, \mathcal{S})$ has fellow projections but does not have bounded projections on $H$ (if $H \neq G$ ) (see Corollary 5.4 and Theorem 5.5). This is the first known example of a triple $(G, \mathcal{S}, H)$ where $(G, \mathcal{S})$ has the falsification by fellow 
traveller property, has fellow projections on $H$, and does not have bounded projections on $H$. On the other hand, note that, by the results of Section 4, the coset growth series $\mathrm{Gr}_{G, H, \mathcal{S}}(t)$ is rational in our case, and we do not know if there exists such an example with a non-rational coset growth series. Note also that the transversal $T$ defined and studied in Section 3 is an important tool in the proof of Theorem 5.5.

\section{Preliminaries}

Let $G$ be a Garside group with Garside structure $(G, M, \Delta)$. Recall that $\mathcal{S}=\operatorname{Div}(\Delta) \backslash$ $\{1\}$ is the generating system of $G$ that will be considered in the present paper and $\lg =\lg _{\mathcal{S}}$ denotes the word length with respect to $\mathcal{S}$. The left greedy normal form of an element $a \in M$ is defined to be the unique expression $a=u_{1} u_{2} \cdots u_{p}$ of $a$ over $\mathcal{S}$ such that $u_{p} \neq 1$ and $\left(u_{i} \cdots u_{p}\right) \wedge_{L} \Delta=u_{i}$ for all $i \in\{1, \ldots, p\}$. We define the right greedy normal form of $a$ in a similar way. The following two theorems contain fundamental results on Garside groups.

Theorem 2.1 (Dehornoy-Paris [12], Dehornoy [9]) (1) Let $a \in M$, let $a=u_{1}$ $u_{2} \cdots u_{p}$ be its left greedy normal form and let $a=u_{q}^{\prime} \cdots u_{2}^{\prime} u_{1}^{\prime}$ be its right greedy normal form. Then $p=q=\lg (a)$.

(2) Let $\alpha \in G$. There exists a unique pair $(a, b) \in M \times M$ such that $\alpha=b^{-1} a$ and $a \wedge_{L} b=1$. Similarly, there exists a unique pair $\left(a^{\prime}, b^{\prime}\right) \in M \times M$ such that $\alpha=a^{\prime} b^{-1}$ and $a^{\prime} \wedge_{R} b^{\prime}=1$. In this case we have $\lg (\alpha)=\lg (a)+\lg (b)=$ $\lg \left(a^{\prime}\right)+\lg \left(b^{\prime}\right)$.

The expressions $\alpha=b^{-1} a$ and $\alpha=a^{\prime} b^{-1}$ given in Theorem 2.1 (2) are called left orthogonal form of $\alpha$ and right orthogonal form of $\alpha$, respectively. Let $\alpha \in G$ and let $\alpha=b^{-1} a$ be its left orthogonal form. Let $a=u_{1} u_{2} \cdots u_{p}$ and $b=v_{1} v_{2} \cdots v_{q}$ be the left greedy normal forms of $a$ and $b$, respectively. Then the expression $\alpha=$ $v_{q}^{-1} \cdots v_{2}^{-1} v_{1}^{-1} u_{1} u_{2} \cdots u_{p}$ is called the left greedy normal form of $\alpha$. Note that, by Theorem $2.1, \lg (\alpha)=p+q$. We define the right greedy normal form of an element of $G$ in a similar way.

An element $a \in M$ is called unmovable if $\Delta \not_{L} a$ or, equivalently, if $\Delta \not_{R} a$. On the other hand, we denote by $\Phi: G \rightarrow G, \alpha \mapsto \Delta \alpha \Delta^{-1}$, the conjugation by $\Delta$. Note that $\Phi(\mathcal{S})=\mathcal{S}$ and $\Phi(M)=M$, and therefore $\Phi$ preserves $\leq_{L}$ and $\leq_{R}$ and thus, it commutes with $\wedge_{L}, \vee_{L}, \wedge_{R}$ and $\vee_{R}$. 
Theorem 2.2 (Dehornoy-Paris [12], Dehornoy [9]) Let $\alpha \in G$. There exists a unique pair $(a, k) \in M \times \mathbb{Z}$ such that $a$ is unmovable and $\alpha=\Delta^{k} a$. Similarly, there exists a unique pair $\left(a^{\prime}, k^{\prime}\right) \in M \times \mathbb{Z}$ such $a^{\prime}$ is unmovable and $\alpha=a^{\prime} \Delta^{k^{\prime}}$, where $k^{\prime}=k$ and $a^{\prime}=\Phi^{k}(a)$.

The expressions $\alpha=\Delta^{k} a$ and $\alpha=a^{\prime} \Delta^{k^{\prime}}$ given in Theorem 2.2 are called the left $\Delta$-form of $\alpha$ and the right $\Delta$-form of $\alpha$, respectively. Note that $a^{\prime} \Delta^{k^{\prime}}=\Phi^{-k}\left(\Delta^{k} a\right)$. We denote by $\sigma: \operatorname{Div}(\Delta) \rightarrow \operatorname{Div}(\Delta)$ the map such that $\Delta=u \sigma(u)$ for all $u \in \operatorname{Div}(\Delta)$. Note that $\sigma(\Delta)=1$ and $\sigma(1)=\Delta$. The following is proved in Picantin [23, Lemma 5.1].

Proposition 2.3 (Picantin [23]) Let $\alpha \in G$ and let $\alpha=v_{q}^{-1} \cdots v_{2}^{-1} v_{1}^{-1} u_{1} u_{2} \cdots u_{p}$ be its left greedy normal form.

(1) Suppose that $q=0$. Let $r \in\{0,1, \ldots, p\}$ such that $u_{r}=\Delta$ and $u_{r+1} \neq \Delta$. Set $c=u_{r+1} \cdots u_{p}$. Then the left $\Delta$-form of $\alpha$ is $\alpha=\Delta^{r} c$, and the left greedy normal form of $c$ is $c=u_{r+1} \cdots u_{p}$.

(2) Suppose that $p=0$. Let $r \in\{0,1, \ldots, q\}$ such that $v_{r}=\Delta$ and $v_{r+1} \neq \Delta$. For each $i \in\{r+1, \ldots, q\}$ we set $w_{i}=\Phi^{i}\left(\sigma\left(v_{i}\right)\right)$. Let $c=w_{q} \cdots w_{r+1}$. Then the left $\Delta$-form of $\alpha$ is $\alpha=\Delta^{-q} c$, and the left greedy normal form of $c$ is $c=w_{q} \cdots w_{r+1}$.

(3) Suppose that $p \geq 1$ and $q \geq 1$. For each $i \in\{1, \ldots, q\}$ we set $w_{i}=\Phi^{i}\left(\sigma\left(v_{i}\right)\right)$. Let $c=w_{q} \cdots w_{1} u_{1} \cdots u_{p}$. Then the left $\Delta$-form of $\alpha$ is $\alpha=\Delta^{-q} c$, and the left greedy normal form of $c$ is $c=w_{q} \cdots w_{1} u_{1} \cdots u_{p}$.

Each of the cases of the proposition above correspond to each of the cases of the corollary below.

Corollary 2.4 Let $\alpha \in G$ and let $\alpha=\Delta^{p} a$ be its left $\Delta$-form.

(1) If $p \geq 0$, then $\lg (\alpha)=\lg (a)+p$.

(2) If $p \leq-\lg (a)$, then $\lg (\alpha)=-p$.

(3) If $-\lg (a) \leq p \leq 0$, then $\lg (\alpha)=\lg (a)$.

Summarizing $\lg (\alpha)=\max (\lg (a)+p,-p, \lg (a))$.

The following theorem contains fundamental results on parabolic subgroups of Garside groups.

Theorem 2.5 (Godelle [17]) Let $(H, N, \delta)$ be a parabolic substructure of $(G, M, \Delta)$. 
(1) $N=H \cap M$.

(2) $H$ is a Garside group with Garside structure $(H, N, \delta)$.

(3) Let $a \in N$, let $a=u_{1} u_{2} \cdots u_{p}$ be its left greedy normal form, and let $a=$ $u_{p}^{\prime} \cdots u_{2}^{\prime} u_{1}^{\prime}$ be its right greedy normal form with respect to $(G, M, \Delta)$. Then $u_{i}, u_{i}^{\prime} \in \operatorname{Div}(\delta)$ for all $i \in\{1, \ldots, p\}, a=u_{1} u_{2} \cdots u_{p}$ is the left greedy normal form of a with respect to $(H, N, \delta)$, and $a=u_{p}^{\prime} \cdots u_{2}^{\prime} u_{1}^{\prime}$ is its right greedy normal form with respect to $(H, N, \delta)$.

(4) Let $\alpha, \beta \in H$ and $\gamma \in G$. If $\alpha \leq_{L} \gamma \leq_{L} \beta$, then $\gamma \in H$. Similarly, if $\alpha \leq_{R} \gamma \leq_{R} \beta$, then $\gamma \in H$.

(5) Let $\alpha, \beta \in H$. Then $\alpha \wedge_{L} \beta, \alpha \vee_{L} \beta \in H$ and $\alpha \wedge_{R} \beta, \alpha \vee_{R} \beta \in H$.

(6) Let $\alpha \in H$ and let $\alpha=b^{-1} a$ (resp. $\alpha=a^{\prime} b^{-1}$ ) be its left orthogonal form (resp. be its right orthogonal form) with respect to $(G, M, \Delta)$. Then $a, b \in N$ (resp. $a^{\prime}, b^{\prime} \in N$ ) and $\alpha=b^{-1} a$ (resp. $\alpha=a^{\prime} b^{-1}$ ) is the left orthogonal form (resp. the right orthogonal form) of $\alpha$ with respect to $(H, N, \delta)$.

The following fact will be used frequently.

Remark Let $(H, N, \delta)$ be a parabolic substructure of $(G, M, \Delta)$. Let $a, b \in M$ such that $a b \in N$, then $a, b \in N$. Indeed, since $a b \in N, 1 \leq_{L} a \leq_{L} a b$ and by the above $a \in H \cap M=N$. Similarly, $b \leq_{R} a b$ and $1 \leq_{R} b \leq_{R} a b$ and therefore $b \in H \cap M=N$.

\section{Transversal}

$>$ From now on we fix a Garside group $G$ with Garside structure $(G, M, \Delta)$ and a parabolic substructure $(H, N, \delta)$. Our goal in the present section is to define a set $T$, to show that $T$ is a transversal of $H$ in $G$, and to show that $\lg (\theta)=\lg (H \theta)$ for all $\theta \in T$. In order to define the set $T$ we need the following.

Lemma 3.1 (Dehornoy [10]) Let $a \in M$. There exists a unique $b \in N$ such that $\left\{c \in N \mid c \leq_{L} a\right\}=\left\{c \in N \mid c \leq_{L} b\right\}$.

The element $b$ of Lemma 3.1 is called the $N$-tail of $a$ and is denoted by $b=\tau_{N}(a)=$ $\tau(a)$. We say that $a$ is $N$-reduced if $\tau_{N}(a)=1$. Note that $a$ is $N$-reduced if and only if $a \wedge_{L} b=1$ for all $b \in N$, or, equivalently, if and only if $a \wedge_{L} \delta=1$. On the other hand we set $\omega=\delta^{-1} \Delta \in M$. This element will play a key role in our study. Recall that $\Phi: G \rightarrow G, \alpha \mapsto \Delta \alpha \Delta^{-1}$, denotes the conjugation by $\Delta$. Furthermore, we denote by 
$\varphi: H \rightarrow H, \beta \mapsto \delta \beta \delta^{-1}$, the conjugation by $\delta$ in $H$. Note that $\omega^{-1} \beta \omega=\left(\Phi^{-1} \circ \varphi\right)(\beta)$ for all $\beta \in H$. In particular, $\omega^{-1} N \omega=\Phi^{-1}(N) \subset M$.

Let $\alpha \in G$ and let $\alpha=a \Delta^{p}$ be its right $\Delta$-form. We say that $\alpha$ is $(H, N)$-reduced if $a$ is $N$-reduced and either $p=0$ or $\left(p<0\right.$ and $\left.\omega \not_{L} a\right)$. Define $T$ to be the set of $(H, N)$-reduced elements of $G$. The purpose of the present section is to prove the following two theorems.

Theorem 3.2 The set $T$ is a right transversal of $H$ in $G$.

Theorem 3.3 We have $\lg (\theta)=\lg (H \theta)$ for all $\theta \in T$.

The following three lemmas are preliminaries to the proofs of Theorem 3.2 and Theorem 3.3 .

Lemma 3.4 Let $b \in N$. Then $b \wedge_{L} \omega=1$ and $b \vee_{L} \omega=b \omega=\omega b^{\prime}$, where $b^{\prime}=\left(\Phi^{-1} \circ \varphi\right)(b)$.

Proof Let $x=b \wedge_{L} \omega$. First observe that $1 \leq_{L} x \leq_{L} b$ and hence $x \in N$ and $\delta x \in$ $N \subseteq M$. Since $x \leq_{L} \omega$, we have $\delta x \leq_{L} \delta \omega=\Delta$, hence $\delta x \in \operatorname{Div}(\Delta) \cap N=\operatorname{Div}(\delta)$. As $\delta x \in \operatorname{Div}(\delta)$ implies that $x^{-1} \in N$, we obtain that $x \in N \cap N^{-1}=\{1\}$. This finish the proof of the first claim.

Now, let $y=b \vee_{L} \omega$. As previously observed, $b^{\prime}=\left(\Phi^{-1} \circ \varphi\right)(b)=\omega^{-1} b \omega$, hence $b \omega=\omega b^{\prime}$. In particular, $b, \omega \leq_{L} b \omega=\omega b^{\prime}$ and hence $y \leq_{L} b \omega=\omega b^{\prime}$. Let $y_{1}^{\prime} \in M$ such that $\omega y_{1}^{\prime}=y$. Since $y \leq_{L} \omega b^{\prime}$, we have $y_{1}^{\prime} \leq_{L} b^{\prime}$, hence there exists $y_{2}^{\prime} \in M$ such that $y_{1}^{\prime} y_{2}^{\prime}=b^{\prime}$. We have $b^{\prime} \in \Phi^{-1}(N)$ and $\Phi^{-1}(N)$ is a parabolic submonoid, hence $y_{1}^{\prime}, y_{2}^{\prime} \in \Phi^{-1}(N)$. Let $y_{1}=\left(\varphi^{-1} \circ \Phi\right)\left(y_{1}^{\prime}\right)$ and $y_{2}=\left(\varphi^{-1} \circ \Phi\right)\left(y_{2}^{\prime}\right)$. Then $y_{1}, y_{2} \in N$ and $b=y_{1} y_{2}$. We have $b=y_{1} y_{2} \leq_{L} y=\omega y_{1}^{\prime}=y_{1} \omega$, hence $y_{2} \leq_{L} \omega$. Since $y_{2} \in N$ and, by the above, $y_{2} \wedge_{L} \omega=1$, it follows that $y_{2}=1$, hence $y_{1}=b$, and therefore $y=b \omega$.

The following is a direct consequence of Dehornoy-Paris [12, Lemma 8.6, Lemma 8.7].

Lemma 3.5 Let $a, b_{1}, b_{2} \in M$. Then $\lg \left(b_{1} a b_{2}\right) \geq \lg (a)$.

Lemma 3.6 Let $c$ be a $N$-reduced element in $M$, let $k \geq 0$ be an integer, and let $d=\omega_{1} \omega_{2} \cdots \omega_{k} \Phi^{-k}(c)$, where $\omega_{i}=\Phi^{-i+1}(\omega)$ for all $i \in\{1, \ldots, k\}$. Then $d$ is $N$-reduced. 
Remark Let $k \geq 0$ and let $\omega_{i}=\Phi^{-i+1}(\omega)$ for all $i \in\{1, \ldots, k\}$ as in the above statement. Then $\omega_{1} \cdots \omega_{k}=\delta^{-k} \Delta^{k}$. This equality will be often used in the remainder of the paper.

Proof We argue by induction on $k$. The case $k=0$ being trivial, we can assume that $k \geq 1$ and that the inductive hypothesis holds. Note that, if $k \geq 1$, then $\omega \leq_{L} d$. Let $b \in N$ such that $b \leq_{L} d$. In particular, $b \vee_{L} \omega \leq d$. By Lemma 3.4, $b \vee_{L} \omega=$ $\omega\left(\Phi^{-1} \circ \varphi\right)(b) \leq_{L} \omega_{1} \omega_{2} \cdots \omega_{k} \Phi^{-k}(c)$, hence $\left(\Phi^{-1} \circ \varphi\right)(b) \leq_{L} \omega_{2} \cdots \omega_{k} \Phi^{-k}(c)$, and therefore $\varphi(b) \leq_{L} \omega_{1} \cdots \omega_{k-1} \Phi^{-k+1}(c)$. Recall that $\varphi(N)=N$. By induction it follows that $\varphi(b)=1$, hence $b=1$.

Proof of Theorem 3.2 We start by showing that each right-coset $C$ of $H$ in $G$ contains an element of $T$. Suppose first that $C$ contains an element $a \in M$. Let $c \in M$ such that $a=\tau(a) c$. Then $c \in T$ and $C=H a=H c$. Now, we assume that $C$ contains no element of $M$ and note that elements not in $M$ have right $\Delta$-form $a \Delta^{-p}$ with $p \geq 1$. Let $\alpha \in C$ and let $\alpha=a \Delta^{-p}$ be the right $\Delta$-form of $\alpha$. We choose $\alpha$ so that $p$ is minimal. Let $c \in M$ such that $a=\tau(a) c$. Set $\theta=c \Delta^{-p}$. Since $C=H \alpha=H \theta$, it suffices to show that $\theta \in T$. The element $c$ is unmovable since $c \leq_{R} a$ and $a$ is unmovable. Furthermore, $c$ is $N$-reduced since $a=\tau(a) c$. Suppose that $\omega \leq_{L} c$. Let $c^{\prime} \in M$ such that $c=\omega c^{\prime}$. Then $\delta \theta=\delta \omega c^{\prime} \Delta^{-p}=\Phi\left(c^{\prime}\right) \Delta^{-p+1} \in H \theta=C$, which contradicts the minimality of $p$. So, $\omega \not_{L} c$, thus $\theta=c \Delta^{-p} \in T$.

Now, we take two elements $\theta_{1}, \theta_{2} \in T$, we suppose that there exists $\beta \in H$ such that $\beta \theta_{1}=\theta_{2}$, and we prove that $\beta=1$ and $\theta_{1}=\theta_{2}$. Let $\theta_{1}=c_{1} \Delta^{-p_{1}}$ and $\theta_{2}=c_{2} \Delta^{-p_{2}}$ be the right $\Delta$-forms of $\theta_{1}$ and $\theta_{2}$, respectively. By definition, $c_{i}$ is $N$-reduced, $p_{i} \geq 0$, and $\omega \not_{L} c_{i}$ if $p_{i} \geq 1$, for $i \in\{1,2\}$. We can assume without loss of generality that $p_{1} \geq p_{2}$. Let $\beta=b_{2}^{-1} b_{1}$ be the left orthogonal form of $\beta$. Then $b_{1} c_{1}=b_{2} c_{2} \Delta^{p_{1}-p_{2}}$. Suppose first that $p_{1}-p_{2}>0$. In particular, $p_{1}>0$, hence $\omega \leq_{L} c_{1}$. Since $\Delta \leq_{R} b_{1} c_{1}$ in this case, we have $\Delta \leq_{L} b_{1} c_{1}$, hence $\omega \leq_{L} b_{1} c_{1}$, thus, by Lemma 3.4, $\omega \vee_{L} b_{1}=b_{1} \omega \leq_{L} b_{1} c_{1}$, and therefore $\omega \leq_{L} c_{1}$ : contradiction. So, $p_{1}=p_{2}$ and $b_{1} c_{1}=b_{2} c_{2}$. Let $y=b_{1} \vee_{L} b_{2}$. We have $y \in N$, since $b_{1}, b_{2} \in N$ and $N$ is a parabolic submonoid. Let $y_{1} \in M$ such that $y=b_{1} y_{1}$. Here again, $y_{1} \in N$, since $N$ is a parabolic submonoid. We have $y=b_{1} y_{1} \leq_{L} b_{1} c_{1}$, since $b_{1} c_{1}=b_{2} c_{2}$, hence $y_{1} \leq{ }_{L} c_{1}$. It follows that $y_{1}=1$, since $c_{1}$ is $N$-reduced, hence $y=b_{1}$. Similarly, $y=b_{2}$, hence $b_{1}=b_{2}=1$, because $b_{1} \wedge_{L} b_{2}=1$. So, $\beta=1$, and $\theta_{1}=\theta_{2}$.

Given $\theta \in T$ and $\beta \in H$ we will need to understand $\beta \theta$. The following three lemmas are the cases for $\theta \in T$, and $\beta \in H$ that will appear in the proofs of Theorems 3.3 and 5.5 . 
Lemma 3.7 Let $c \in T \cap M$ and $\beta \in H$. Let $\beta=b_{2}^{-1} b_{1}$ be the left orthogonal form of $\beta$. Then $1=b_{2} \wedge_{L}\left(b_{1} c\right)$ and therefore $b_{2}^{-1}\left(b_{1} c\right)$ is the left orthogonal form of $\beta \theta$.

Proof Recall that $b_{1}, b_{2} \in N$, since $H$ is a parabolic subgroup. Set $y_{1}=b_{2} \wedge_{L}\left(b_{1} c\right)$. We have $y_{1} \in N$ since $y_{1} \leq_{L} b_{2}$. Let $y_{2}=b_{1} \vee_{L} y_{1}$. We have $y_{2} \in N$ since $y_{1}, b_{1} \in N$. Moreover, we have $y_{2} \leq_{L} b_{1} c$ since $b_{1} \leq_{L} b_{1} c$ and $y_{1} \leq_{L} b_{1} c$. Let $y_{3} \in M$ such that $y_{2}=b_{1} y_{3}$. We have $y_{3} \in N$ (since $y_{2} \in N$ ), $y_{3} \leq_{L} c$ and $c$ is $N$-reduced, hence $y_{3}=1$. So, $y_{2}=b_{1}$, which implies that $y_{1} \leq_{L} b_{1}$. We also have $y_{1} \leq_{L} b_{2}$ and $b_{1} \wedge_{L} b_{2}=1$, hence $y_{1}=1$.

Lemma 3.8 Let $\theta=c \Delta^{-p} \in T \backslash M$ in right $\Delta$-form with $p \geq 1$ and $\beta=b \in N$. Then $b c$ is unmovable and $b c \Delta^{-p}$ is the right $\Delta$-form of $\beta \theta$.

Proof Notice that $c$ is unmovable and $N$-reduced, $\omega \mathbb{L}_{L} c$, and $p \geq 1$. If we had $\Delta \leq_{L} b c$, then we would have $\omega \leq_{L} b c$, hence, by Lemma 3.4, we would have $b \vee_{L} \omega=b \omega \leq_{L} b c$, and therefore we would have $\omega \leq_{L} c$ : contradiction. So, $b c$ is unmovable.

Lemma 3.9 Let $\theta=c \Delta^{-p} \in T \backslash M$ in right $\Delta$-form with $p \geq 1$ and $\beta=b \delta^{-k} \in H \backslash N$ in right $\delta$-form with $k \geq 1$. Then the right $\Delta$-form of $\beta \theta$ is $b \omega_{1} \ldots \omega_{k} \Phi^{-k}(c) \Delta^{-k-p}$, where $\omega_{i}=\Phi^{-i+1}$ for all $i \in\{1, \ldots, k\}$.

Proof As $\theta \in T, c$ is unmovable and $N$-reduced, $\omega \mathbb{L}_{L} c$, and $p \geq 1$. By using the equality $\delta^{-1}=\omega \Delta^{-1}$ it is easily seen that $\beta \theta=b \omega_{1} \omega_{2} \cdots \omega_{k} \Phi^{-k}(c) \Delta^{-p-k}$, where $\omega_{i}=\Phi^{-i+1}(\omega)$ for all $i \in\{1, \ldots, k\}$. By Lemma 3.6 we have $\tau\left(b \omega_{1} \cdots \omega_{k} \Phi^{-k}(c)\right)=$ $b$. Thus, if $\Delta \leq_{L} b \omega_{1} \cdots \omega_{k} \Phi^{-k}(c)$, then $\delta \leq_{L} b \omega_{1} \cdots \omega_{k} \Phi^{-k}(c)$, hence $\delta \leq_{L} b$ : contradiction. So, $\Delta \not_{L} b \omega_{1} \cdots \omega_{k} \Phi^{-k}(c)$.

Proof of Theorem 3.3 We take $\theta \in T$ and $\beta \in H$ and we show that $\lg (\beta \theta) \geq \lg (\theta)$. Suppose first that $\theta=c \in M$. Let $\beta=b_{2}^{-1} b_{1}$ be the left orthogonal form of $\beta$. Lemma 3.7 imples that $b_{2}^{-1}\left(b_{1} c\right)$ is the left orthogonal form of $\beta \theta$. By applying Theorem 2.1 and Lemma 3.5 we finally obtain $\lg (\beta \theta)=\lg \left(b_{2}\right)+\lg \left(b_{1} c\right) \geq \lg (c)=\lg (\theta)$.

Assume that $\theta \notin M$ and $\beta=b \in N$. We write $\theta$ in the form $\theta=c \Delta^{-p}$, where $c$ is unmovable and $N$-reduced, $\omega \mathbb{Z}_{L} c$, and $p \geq 1$. By Lemma $3.8 b c$ is unmovable and $b c \Delta^{-p}$ is the right $\Delta$-form of $\beta \theta$. By Corollary 2.4 it follows that $\lg (\beta \theta)=$ $\max (\lg (b c), p) \geq \max (\lg (c), p)=\lg (\theta)$.

Assume that $\theta \notin M$ and $\beta \notin N$. As before, we write $\theta$ in the form $\theta=c \Delta^{-p}$, where $c$ is unmovable and $N$-reduced, $\omega \mathbb{L}_{L} c$, and $p \geq 1$. On the other hand we write $\beta$ in the 
form $\beta=b \delta^{-k}$, where $b \in N, \delta \not_{L} b$, and $k \geq 1$. By Lemma 3.9, the right $\Delta$-form of $\beta \theta$ is $b \omega_{1} \cdots \omega_{k} \Phi^{-k}(c) \Delta^{-k-p}$, where $\omega_{i}=\Phi^{-i+1}$ for all $i \in\{1, \ldots, k\}$. By Corollary 2.4 and Lemma 3.5 it follows that $\lg (\beta \theta)=\max \left(\lg \left(b \omega_{1} \cdots \omega_{k} \Phi^{-k}(c)\right), p+k\right) \geq$ $\max (\lg (c), p)=\lg (\theta)$.

\section{Regular language}

A finite state automaton is defined to be a quintuple $\mathcal{F}=\left(X, \mathcal{A}, \mu, Y, x_{0}\right)$, where $X$ is a finite set, called the set of states, $\mathcal{A}$ is a finite set, called the alphabet, $\mu: X \times \mathcal{A} \rightarrow X$ is a function, called the transition function, $Y$ is a subset of $X$, called the set of accepted states, and $x_{0}$ is an element of $X$, called the initial state. For $x \in X$ and $U=u_{1} u_{2} \cdots u_{p} \in \mathcal{A}^{*}$ we define $\mu(x, U) \in X$ by induction on $p$ as follows.

$$
\mu(x, U)= \begin{cases}x & \text { if } p=0 \\ \mu\left(\mu\left(x, u_{1} \cdots u_{p-1}\right), u_{p}\right) & \text { if } p \geq 1\end{cases}
$$

Then the set $L_{\mathcal{F}}=\left\{U \in \mathcal{A}^{*} \mid \mu\left(x_{0}, U\right) \in Y\right\}$ is called the language recognized by $\mathcal{F}$. A regular language is a language recognized by a finite state automaton.

Recall that $G$ is a given Garside group with Garside structure $(G, M, \Delta)$, that $(H, N, \delta)$ is a parabolic substructure, and that $T$ is the transversal of $H$ in $G$ defined in Section 3. The following is used to understand the language defined by the automaton below. Recall that $\sigma: \operatorname{Div}(\Delta) \rightarrow \operatorname{Div}(\Delta)$ is the function such that $\Delta=u \sigma(u)$ for all $u \in \operatorname{Div}(\Delta)$.

Theorem 4.1 (Dehornoy-Paris [12]) Let $u_{1}, \ldots, u_{p}, v_{1}, \ldots, v_{q} \in \mathcal{S}$. Then $v_{q}^{-1} \ldots$ $v_{2}^{-1} v_{1}^{-1} u_{1} u_{2} \cdots u_{p}$ is a left greedy normal form if and only if $\sigma\left(u_{i}\right) \wedge_{L} u_{i+1}=1$ for all $i \in\{1, \ldots, p-1\}, \sigma\left(v_{j}\right) \wedge_{L} v_{j+1}=1$ for all $j \in\{1, \ldots, q-1\}$, and $u_{1} \wedge_{L} v_{1}=1$.

We define a finite state automaton $\mathcal{F}=\left(X, \mathcal{A}, \mu, Y, x_{0}\right)$ as follows. We set $\mathcal{A}=$ $\mathcal{S} \cup \mathcal{S}^{-1}, X=\mathcal{A} \cup\left\{x_{0}, x_{1}\right\}, Y=\mathcal{A} \cup\left\{x_{0}\right\}$, and we define $\mu: X \times \mathcal{A} \rightarrow X$ as follows. 
Let $u, v \in \mathcal{S}$.

$$
\begin{aligned}
& \mu\left(x_{0}, v\right)=\left\{\begin{array}{ll}
v & \text { if } v \wedge_{L} \delta=1, \\
x_{1} \quad \text { otherwise, }
\end{array} \quad \mu\left(x_{0}, v^{-1}\right)= \begin{cases}v^{-1} & \text { if } v=\Delta, \\
v^{-1} & \text { if } \sigma(v) \wedge_{L} \delta=1 \\
& \text { and } \omega \not_{L} \sigma(v), \\
x_{1} & \text { otherwise },\end{cases} \right. \\
& \mu(u, v)= \begin{cases}v & \text { if } \sigma(u) \wedge_{L} v=1, \quad \mu\left(u, v^{-1}\right)=x_{1}, \\
x_{1} & \text { otherwise, }\end{cases} \\
& \mu\left(u^{-1}, v\right)=\left\{\begin{array}{ll}
v & \text { if } u \wedge_{L} v=1, \\
x_{1} & \text { otherwise, }
\end{array} \quad \mu\left(u^{-1}, v^{-1}\right)= \begin{cases}v^{-1} & \text { if } \sigma(v) \wedge_{L} u=1, \\
x_{1} & \text { otherwise },\end{cases} \right. \\
& \mu\left(x_{1}, v\right)=\mu\left(x_{1}, v^{-1}\right)=x_{1} .
\end{aligned}
$$

Recall that ev : $\mathcal{A}^{*} \rightarrow G$ is the map that sends each word $U$ to the element of $G$ that it represents. The purpose of the present section is to prove the following.

Theorem 4.2 We have $\operatorname{ev}\left(L_{\mathcal{F}}\right)=T$, the restriction ev $: L_{\mathcal{F}} \rightarrow T$ is a bijective correspondence, and $\lg (U)=\lg (\operatorname{ev}(U))$ for all $U \in L_{\mathcal{F}}$.

Recall that for each $n \in \mathbb{N}$ we denote by $e(n)=e_{G, H, \mathcal{S}}(n)$ the number of right cosets of $H$ in $G$ of length $n$. Recall also that the coset growth series of $H$ in $G$ is $\operatorname{Gr}_{G, H, \mathcal{S}}(t)=\sum_{n=0}^{\infty} e(n) t^{n}$. By Theorem 3.2, Theorem 3.3 and Theorem $4.2 e(n)$ is equal to the number of words of length $n$ in the regular language $L_{\mathcal{F}}$. In other words, $\mathrm{Gr}_{G, H, \mathcal{S}}(t)$ is the growth series of $L_{\mathcal{F}}$. Since we know that the growth series of a regular language is rational (see Flajolet-Sedgewick [15], for example), it follows that:

Corollary 4.3 The formal series $\mathrm{Gr}_{G, H, \mathcal{S}}(t)$ is rational.

Proof of Theorem 4.2 Set $T^{\prime}=\operatorname{ev}\left(L_{\mathcal{F}}\right)$. By Theorem 4.1 the restriction ev : $L_{\mathcal{F}} \rightarrow$ $T^{\prime}$ is a bijective correspondence, and $T^{\prime}$ is the set of elements $\theta=v_{q}^{-1} \cdots v_{2}^{-1} v_{1}^{-1} u_{1} u_{2}$ $\cdots u_{p}$ written in left greedy normal form such that:

- if $q=0$ and $p \geq 1$, then $u_{1} \wedge_{L} \delta=1$,

- if $q \geq 1$, then either $v_{q}=\Delta$ or $\left(\sigma\left(v_{q}\right) \wedge_{L} \delta=1\right.$ and $\left.\omega \not_{L} \sigma\left(v_{q}\right)\right)$.

Moreover, by Theorem 2.1, we have $\lg (U)=\lg (\operatorname{ev}(U))$ for all $U \in L_{\mathcal{F}}$. So, it remains to show that $T^{\prime}=T$.

Let $\theta=v_{q}^{-1} \cdots v_{2}^{-1} v_{1}^{-1} u_{1} u_{2} \cdots u_{p}$ be an element of $G$ written in left greedy normal form. Suppose first that $q=0$. Then $\theta=u_{1} u_{2} \cdots u_{p}$. If $p=0$, then $\theta=1 \in T \cap T^{\prime}$. 
So, we can assume that $p \geq 1$. Since $\delta \in \operatorname{Div}(\Delta)$, we have $\theta \wedge_{L} \delta=\left(\theta \wedge_{L} \Delta\right) \wedge_{L} \delta=$ $u_{1} \wedge_{L} \delta$, hence

$$
\theta \in T \Leftrightarrow \theta \wedge_{L} \delta=1 \Leftrightarrow u_{1} \wedge_{L} \delta=1 \Leftrightarrow \theta \in T^{\prime} .
$$

Suppose that $p=0$ and $q \geq 1$. Then $\theta=v_{q}^{-1} \cdots v_{2}^{-1} v_{1}^{-1}$. Let $r \in\{0,1, \ldots, q\}$ such that $v_{i}=\Delta$ for all $i \in\{1, \ldots, r\}$ and $v_{r+1} \neq \Delta$. Set $w_{j}=\Phi^{-j+1}\left(\sigma\left(v_{q-j+1}\right)\right)$ for $j \in\{1, \ldots, q-r\}$ and $c=w_{1} \cdots w_{q-r}$. Then, by Proposition 2.3 and Theorem 2.2, $\theta=c \Delta^{-q}, c$ is unmovable, and $c=w_{1} \cdots w_{q-r}$ is the left greedy normal form of $c$. Notice that $w_{1}=\sigma\left(v_{q}\right)$. If $v_{q}=\Delta$, then $c=1$ and $\theta=\Delta^{-q} \in T \cap T^{\prime}$. So, we can assume that $v_{q} \neq \Delta$, that is, $q>r$ and $c \neq 1$. As above, since $\delta \in \operatorname{Div}(\Delta)$, we have $c \wedge_{L} \delta=w_{1} \wedge_{L} \delta=\sigma\left(v_{q}\right) \wedge_{L} \delta$. On the other hand, since $\omega \in \operatorname{Div}(\Delta)$, we have $\omega \leq_{L} c$ if and only if $\omega \leq_{L} w_{1}=\sigma\left(v_{q}\right)$. So,

$\theta \in T \Leftrightarrow\left(c \wedge_{L} \delta=1\right.$ and $\left.\omega \not_{L} c\right) \Leftrightarrow\left(\sigma\left(v_{q}\right) \wedge_{L} \delta=1\right.$ and $\left.\omega \not_{L} \sigma\left(v_{q}\right)\right) \Leftrightarrow \theta \in T^{\prime}$.

Suppose that $p \geq 1$ and $q \geq 1$. Then $\theta=v_{q}^{-1} \cdots v_{2}^{-1} v_{1}^{-1} u_{1} u_{2} \cdots u_{p}$. Since $u_{1} u_{2} \cdots u_{p} \wedge_{L} v_{1} v_{2} \cdots v_{q}=1$, we have $u_{i} \neq \Delta$ for all $i \in\{1, \ldots, p\}$ and $v_{j} \neq \Delta$ for all $j \in\{1, \ldots, q\}$. Set $w_{j}=\Phi^{-j+1}\left(\sigma\left(v_{q-j+1}\right)\right)$ for all $j \in\{1, \ldots, q\}$ and $w_{j}=\Phi^{-q}\left(u_{j-q}\right)$ for all $j \in\{q+1, \ldots, q+p\}$. Let $c=w_{1} \cdots w_{q+p}$. Then, by Proposition 2.3 and Theorem 2.2, $\theta=c \Delta^{-q}, c$ is unmovable, and $c=w_{1} \cdots w_{q+p}$ is the left greedy normal form of $c$. By applying the same reasoning as in the previous case, we then obtain

$\theta \in T \Leftrightarrow\left(c \wedge_{L} \delta=1\right.$ and $\left.\omega \not_{L} c\right) \Leftrightarrow\left(\sigma\left(v_{q}\right) \wedge_{L} \delta=1\right.$ and $\left.\omega \not_{L} \sigma\left(v_{q}\right)\right) \Leftrightarrow \theta \in T^{\prime}$.

\section{Projections}

Let $G$ be a group generated by a finite set $\mathcal{S}$. We set $\mathcal{A}=\mathcal{S} \cup \mathcal{S}^{-1}$ and, as before, we denote by ev : $\mathcal{A}^{*} \rightarrow G$ the map which sends each word $U \in \mathcal{A}^{*}$ to the element of $G$ that it represents. We denote by $\lg =\lg _{\mathcal{S}}$ the word length in $G$ with respect to $\mathcal{S}$ and by $d: G \times G \rightarrow \mathbb{N}$ the distance function induced by $\lg$. Recall that $d(\alpha, \beta)=\lg \left(\alpha^{-1} \beta\right)$ for all $\alpha, \beta \in G$. Recall also that, for $\alpha \in G$ and $X \subset G$, the distance from $\alpha$ to $X$ is $d(\alpha, X)=\min \{d(\alpha, \beta) \mid \beta \in X\}$. The diameter of a subset $X \subset G$ is $\operatorname{diam}(X)=\max \{d(\alpha, \beta) \mid \alpha, \beta \in X\}$.

A word $U \in \mathcal{A}^{*}$ is called a geodesic (or a reduced word) if $\lg (U)=\lg (\operatorname{ev}(U))$. For a word $U=x_{1} x_{2} \cdots x_{\ell}$ of length $\ell$ and $i \in \mathbb{N}$ we set $U(i)=x_{1} \cdots x_{i}$ if $i \leq \ell$ and 
$U(i)=U$ if $i>\ell$. Let $K$ be a positive constant. We say that two words $U, V \in \mathcal{A}^{*}$ $K$-fellow travel if $d(\operatorname{ev}(U(i)), \operatorname{ev}(V(i))) \leq K$ for all $i \in \mathbb{N}$. We say that $(G, \mathcal{S})$ has the falsification by $K$-fellow traveller property if for each non-geodesic word $U \in \mathcal{A}^{*}$ there exists a strictly shorter word $V \in \mathcal{A}^{*}$ such that $\operatorname{ev}(U)=\operatorname{ev}(V)$ and $U, V K$-fellow travel.

Let $H$ be a subgroup of $G$. The projection of an element $\alpha \in G$ in $H$ is defined to be $\pi_{H}(\alpha)=\{\beta \in H \mid d(\alpha, \beta)=d(\alpha, H)\}$. Let $K$ be a positive constant. We say that $(G, \mathcal{S})$ has $K$-fellow projections on $H$ if, for each $\alpha_{1}, \alpha_{2} \in G$ such that $d\left(\alpha_{1}, \alpha_{2}\right)=1$ and each $\beta_{1} \in \pi_{H}\left(\alpha_{1}\right)$, there exists $\beta_{2} \in \pi_{H}\left(\alpha_{2}\right)$ such that $d\left(\beta_{1}, \beta_{2}\right) \leq K$. We say that $(G, \mathcal{S})$ has $K$-bounded projections on $H$ if, for each $\alpha_{1}, \alpha_{2} \in G$ such that $d\left(\alpha_{1}, \alpha_{2}\right)=1$, we have $\operatorname{diam}\left(\pi_{H}\left(\alpha_{1}\right) \cup \pi_{H}\left(\alpha_{2}\right)\right) \leq K$. Note that, if $G$ has $K$-bounded projections on $H$, then $G$ has $K$-fellow projections on $H$.

As pointed out in the introduction, the starting point of our study was the following two theorems.

Theorem 5.1 (Antolín [1]) Let $G$ be a group generated by a finite set $\mathcal{S}$, let $\mathcal{A}=$ $\mathcal{S} \cup \mathcal{S}^{-1}$, and let $H$ be a subgroup of $G$. Assume that $(G, \mathcal{S})$ has the falsification by fellow traveller property and has fellow projections on $H$. Then:

(1) The language $\operatorname{Geo}(H \backslash G, \mathcal{S})=\left\{U \in \mathcal{A}^{*} \mid \lg (U)=\lg (H \operatorname{ev}(U))\right\}$ is regular.

(2) If, furthermore, $(G, \mathcal{S})$ has bounded projections on $H$, then the coset growth series $\mathrm{Gr}_{G, H, \mathcal{S}}(t)$ of $H$ in $G$ is rational.

Theorem 5.2 (Holt [20]) Let $G$ be a Garside group with Garside structure $(G, M, \Delta)$, and let $\mathcal{S}=\operatorname{Div}(\Delta) \backslash\{1\}$. Then $G$ endowed with the generating set $\mathcal{S}$ has the falsification by fellow traveller property.

Let $G$ be a Garside group with Garside structure $(G, M, \Delta)$ and let $(H, N, \delta)$ be a parabolic substructure. So, it is natural to ask whether $(G, \mathcal{S})$ has bounded projections on $H$ and, if not, whether it has fellow projections. The answer is "NO" for the first question (see Corollary 5.4), and is "YES" for the second question (see Theorem 5.5). As pointed out in the introduction, this is the first example we know of a triple $(G, \mathcal{S}, H)$, where $G$ is a group, $\mathcal{S}$ a finite generating set and $H$ is a subgroup of $G$ such that $(G, \mathcal{S})$ has the falsification by fellow traveller property, it has fellow projections on $H$, but it does not have bounded projections on $H$.

$>$ From now on $G$ denotes a given Garside group with Garside structure $(G, M, \Delta)$ and $(H, N, \delta)$ denotes a given parabolic substructure. Recall that $\omega=\delta^{-1} \Delta$, and $\Phi: G \rightarrow G, \alpha \mapsto \Delta \alpha \Delta^{-1}$, denotes the conjugation by $\Delta$. The fact that $G$ does not have bounded projections on $H$ (if $H \neq G$ ) is a direct consequence of the following. 
Lemma 5.3 Suppose that $H \neq G$. Let $k \geq 1$ and let $d_{k}=\omega_{1} \omega_{2} \cdots \omega_{k}$, where $\omega_{i}=\Phi^{-i+1}(\omega)$ for all $i \in\{1, \ldots, k\}$. Then $\operatorname{diam}\left(\pi_{H}\left(d_{k}\right)\right) \geq k$.

Proof By Lemma 3.6 the element $d_{k}$ is $N$-reduced, hence $d_{k} \in T$, and therefore, by Theorem 3.3, $\lg \left(H d_{k}\right)=\lg \left(d_{k}\right)$. It follows that $d\left(d_{k}, H\right)=\lg \left(d_{k}\right), 1 \in \pi_{H}\left(d_{k}\right)$, and $\beta \in \pi_{H}\left(d_{k}\right)$ if and only if $\lg \left(\beta^{-1} d_{k}\right)=\lg \left(d_{k}\right)$ and $\beta \in H$. We have $\delta \Delta=\Delta \sigma(\omega)$, hence $\sigma(\omega)=\Phi^{-1}(\delta)$, and therefore $\sigma\left(\omega_{i}\right)=\Phi^{-i}(\delta)$ for all $i \in\{1, \ldots, k\}$. Let $i \in\{1, \ldots, k-1\}$. Then $\omega_{i+1}=\Phi^{-i}(\omega)$ and, by Lemma 3.4, $\delta \wedge_{L} \omega=1$, hence $\sigma\left(\omega_{i}\right) \wedge_{L} \omega_{i+1}=\Phi^{-i}(\delta) \wedge_{L} \Phi^{-i}(\omega)=1$. It follows by Theorem 4.1 that $\omega_{1} \omega_{2} \cdots \omega_{k}$ is the greedy normal form of $d_{k}$, hence $\lg \left(d_{k}\right)=k$. On the other hand, $\delta^{k} d_{k}=\Delta^{k}$, hence, by Corollary $2.4, \lg \left(\Delta^{k}\right)=k$. Since $\delta^{k} \in H$, this shows that $\delta^{-k} \in \pi_{H}\left(d_{k}\right)$. Finally, by Corollary 2.4, $\delta^{k}$ has length $k$ with respect to $\operatorname{Div}(\delta) \backslash\{1\}$, hence, by Theorem 2.5, $\lg \left(\delta^{-k}\right)=\lg \left(\delta^{k}\right)=k$. So, $\operatorname{diam}\left(\pi_{H}\left(d_{k}\right)\right) \geq k$.

Corollary 5.4 Suppose that $H \neq G$ (and $H \neq 1$ ). Then $G$ does not have bounded projections on $H$.

Proof For each $k \geq 1$ we choose $\alpha_{k} \in G$ such that $d\left(d_{k}, \alpha_{k}\right)=1$. Then, by Lemma 5.3, $\operatorname{diam}\left(\pi_{H}\left(d_{k}\right) \cup \pi_{H}\left(\alpha_{k}\right)\right) \geq \operatorname{diam}\left(\pi_{H}\left(d_{k}\right)\right) \geq k$, hence there is no $K>0$ such that $\operatorname{diam}\left(\pi_{H}\left(d_{k}\right) \cup \pi_{H}\left(\alpha_{k}\right)\right) \leq K$ for all $k \in \mathbb{N}$.

The rest of the section is dedicated to the proof of the following.

Theorem 5.5 The group $G$ has 5 -fellow projections on $H$ with respect to $\mathcal{S}$.

The following three lemmas are preliminaries to the proof of Theorem 5.5. Lemma 5.6 is known to experts but to our knowledge is nowhere explicitly written in the literature.

Lemma 5.6 Let $a, b \in M$ and let $a=u_{m} \cdots u_{2} u_{1}$ and $b=v_{n} \cdots v_{2} v_{1}$ be the right greedy normal forms of $a$ and $b$, respectively. If $a \leq_{R} b$, then $m \leq n$ and for all $i \in\{1, \ldots, m\}$, one has that $u_{i} u_{i-1} \cdots u_{1} \leq_{R} v_{i} v_{i-1} \cdots v_{1}$.

Proof We already know by Theorem 2.1 and Lemma 3.5 that $m=\lg (a) \leq \lg (b)=n$. It remains to show that $u_{i} \cdots u_{1} \leq_{R} v_{i} \cdots v_{1}$ for all $i \in\{1, \ldots, m\}$. We argue by induction on $i$. Note that for $x, y \in M$, if $x \leq_{R} y$ then $\left(x \wedge_{R} \Delta\right) \leq_{R}\left(y \wedge_{R} \Delta\right)$. Therefore, the case $i=1$ is true by definition of a right greedy normal form. So, we can assume that $i \geq 2$ and that the inductive hypothesis holds. By induction we have $u_{i-1} \cdots u_{1} \leq_{R} v_{i-1} \cdots v_{1}$. Let $c \in M$ such that $c u_{i-1} \cdots u_{1}=v_{i-1} \cdots v_{1}$. We have $u_{i} \leq_{R} \Delta, u_{m} \cdots u_{i} \leq_{R} v_{n} \cdots v_{i+1} v_{i} c$ and, by Dehornoy [9, Lemme 3.10], $\left(v_{n} \cdots v_{i+1} v_{i} c\right) \wedge_{R} \Delta=\left(v_{i} c\right) \wedge_{R} \Delta$, hence $u_{i} \leq_{R} v_{i} c$, and therefore $u_{i} u_{i-1} \cdots u_{1} \leq_{R}$ $v_{i} v_{i-1} \cdots v_{1}$. 
Lemma 5.7 Let $a$ be an element of $M$ which can be written in the form $a=$ $\omega_{1} \omega_{2} \cdots \omega_{k} c$, where $\omega_{i}=\Phi^{-i+1}(\omega)$ for all $i \in\{1, \ldots, k\}, \Phi^{-k}(\omega) \not_{L} c$, and $c$ is $\Phi^{-k}(N)$-reduced. Then $\lg (a)=\lg (c)+k$.

Proof We argue by induction on $k$. The case $k=0$ being trivial, we can assume that $k \geq 1$ and that the inductive hypothesis holds. It suffices to show that $a \wedge_{L} \Delta=$ $\omega=\omega_{1}$. Indeed, in that case, by Theorem 2.1, we have $\lg (a)=\lg \left(\omega_{2} \cdots \omega_{k} c\right)+1$. Since $\Phi(\mathcal{S})=\mathcal{S}, \Phi$ preserves $\lg$, thus $\lg \left(\omega_{2} \cdots \omega_{k} c\right)=\lg \left(\omega_{1} \omega_{2} \cdots \omega_{k-1} \Phi(c)\right)$ and $\Phi(c)$ is $\Phi^{-k+1}(N)$-reduced. By induction, $\lg \left(\omega_{2} \cdots \omega_{k} c\right)=\lg (c)+k-1$, hence $\lg (a)=\lg (c)+k$.

Set $x=a \wedge_{L} \Delta$. Since $\omega=\omega_{1} \leq_{L} a$ and $\omega \leq_{L} \Delta$, we have $\omega \leq_{L} x$. Let $x_{1} \in M$ such that $x=\omega x_{1}$. We have $\omega x_{1} \sigma(x)=x \sigma(x)=\Delta=\omega \delta_{1}$, where $\delta_{1}=\Phi^{-1}(\delta)$, hence $x_{1} \leq_{L} \delta_{1}$, thus $x_{1} \in \Phi^{-1}(N)$, since $\Phi^{-1}(N)$ is a parabolic submonoid of $M$. We also have $x_{1} \leq_{L} \omega_{2} \cdots \omega_{k} c$ and $\omega_{2} \cdots \omega_{k} c$ is $\Phi^{-1}(N)$-reduced by Lemma 3.6, hence $x_{1}=1$ and $x=\omega$.

Lemma 5.8 Let $\alpha \in G, \ell=\lg (H \alpha)$ and $\operatorname{Min}(\alpha)=\{\gamma \in H \alpha \mid \lg (\gamma)=\ell\}$. Then $\ell=d(\alpha, H)$ and we have a bijective map $\operatorname{Min}(\alpha) \rightarrow \pi_{H}(\alpha)$ which sends $\gamma$ to $\alpha \gamma^{-1}$ for all $\gamma \in \operatorname{Min}(\alpha)$.

Proof If $\beta \in H$, then $d(\beta, \alpha)=\lg \left(\beta^{-1} \alpha\right) \geq \ell$. On the other hand, if $\gamma \in \operatorname{Min}(\alpha)$ and $\beta=\alpha \gamma^{-1}$, then $\beta \in H$ and $d(\beta, \alpha)=\lg \left(\beta^{-1} \alpha\right)=\lg (\gamma)=\ell$. So, $\ell=d(\alpha, H)$ and $\beta \in \pi_{H}(\alpha)$. Reciprocally, if $\beta \in \pi_{H}(\alpha)$ and $\gamma=\beta^{-1} \alpha \in H \alpha$, then $\lg (\gamma)=$ $d(\beta, \alpha)=\ell$ and $\gamma \in \operatorname{Min}(\alpha)$.

The proof of Theorem 5.5 is divided into three cases, each case being treated in one of the following three lemmas.

Lemma 5.9 Let $\alpha \in G, \beta \in \pi_{H}(\alpha)$ and $u \in \mathcal{S}$. Denote by $\theta$ the element of $T$ such that $H \alpha=H \theta$ and assume that $\theta=c \in M$. Then there exists $\beta^{\prime} \in \pi_{H}(\alpha u)$ such that $d\left(\beta, \beta^{\prime}\right) \leq 3$.

Proof Set $\ell=\lg (H \alpha)$. By Theorem 3.3, $\lg (\theta)=\ell$ and $c=\theta \in \operatorname{Min}(\alpha)$. By Lemma 5.8 there exists $\gamma \in \operatorname{Min}(\alpha)$ such that $\beta=\alpha \gamma^{-1}$. Let $\beta_{1} \in H$ such that $\beta_{1} \theta=\gamma$. Let $b_{1}^{\prime-1} b_{1}$ be the left orthogonal form of $\beta_{1}$. By Lemma 3.7 we have that $b_{1}^{\prime} \wedge_{L}\left(b_{1} c\right)=1$. Thus, by Theorem 2.1, $\ell=\lg (\gamma)=\lg \left(b_{1}^{\prime}\right)+\lg \left(b_{1} c\right)$ and, by Lemma 3.5, $\lg \left(b_{1} c\right) \geq \lg (c)$. Since $\ell=\lg (c)$, it follows that $b_{1}^{\prime}=1, \beta_{1}=b_{1} \in N$ and $\gamma=b_{1} c \in M$. 
Set $b_{2}=\tau_{N}(c u) \in N$ and denote by $c^{\prime}$ the element of $M$ such that $c u=b_{2} c^{\prime}$. Note that $c^{\prime}$ is $N$-reduced, hence $c^{\prime} \in T$. Note also that $H c^{\prime}=H \alpha u$, hence $\theta^{\prime}=c^{\prime}$ is the element of $T$ which lies in $H \alpha u$, and, by Theorem 3.3, $\lg (H \alpha u)=\lg \left(c^{\prime}\right)$. Since $c$ is $N$-reduced, we have $b_{2} \wedge_{L} c=1$, hence, by Theorem 2.1, $\lg \left(b_{2}^{-1} c\right)=\lg \left(b_{2}\right)+\lg (c)$. So,

$\lg (c)+1 \geq \lg (c u)=\lg \left(b_{2} c^{\prime}\right) \geq \lg \left(c^{\prime}\right)=\lg \left(b_{2}^{-1} c u\right) \geq \lg \left(b_{2}^{-1} c\right)-1=\lg \left(b_{2}\right)+\lg (c)-1$, hence $\lg \left(b_{2}\right) \leq 2$. It follows that

$$
\lg \left(c^{\prime}\right) \geq \lg \left(b_{2} c^{\prime}\right)-\lg \left(b_{2}\right) \geq \lg (c u)-2 \geq \lg (c)-2 .
$$

Now we show that there exist $b_{3}, b_{4} \in N$ such that $b_{3} b_{4}=b_{1} b_{2}, \lg \left(b_{4} c^{\prime}\right)=\lg \left(c^{\prime}\right)$ and $\lg \left(b_{3}\right) \leq 3$. Set $n=\lg \left(b_{1} b_{2} c^{\prime}\right)$ and $m=\lg \left(c^{\prime}\right)$. We have

$$
n=\lg \left(b_{1} b_{2} c^{\prime}\right)=\lg \left(b_{1} c u\right) \leq \lg \left(b_{1} c\right)+1=\lg (c)+1 \leq \lg \left(c^{\prime}\right)+3=m+3 .
$$

Let $b_{1} b_{2} c^{\prime}=v_{n} \cdots v_{2} v_{1}$ be the right greedy normal form of $b_{1} b_{2} c^{\prime}$. Then $c^{\prime} \leq_{R}$ $v_{n} \cdots v_{2} v_{1}$. Set $d=v_{m} \cdots v_{2} v_{1}$. Since $\lg \left(c^{\prime}\right)=m$, by Lemma 5.6 we have $c^{\prime} \leq_{R} d$ and, by Theorem 2.1, $m=\lg (d)$. Let $b_{4} \in M$ such that $b_{4} c^{\prime}=d$, and let $b_{3}=v_{n} \cdots v_{m+1}$. We have $b_{3} b_{4} c^{\prime}=b_{1} b_{2} c^{\prime}$, hence $b_{3} b_{4}=b_{1} b_{2}$, therefore $b_{3}, b_{4} \in N$ since $b_{1} b_{2} \in N$ and $N$ is a parabolic submonoid. By the above we also have $\lg \left(b_{3}\right)=n-m \leq 3$ and $\lg \left(b_{4} c^{\prime}\right)=\lg (d)=\lg \left(c^{\prime}\right)$.

Set $\gamma^{\prime}=b_{4} c^{\prime}$ and $\beta^{\prime}=(\alpha u) \gamma^{\prime-1}$. By the above, $\gamma^{\prime} \in \operatorname{Min}(\alpha u)$, hence, by Lemma 5.8, $\beta^{\prime} \in \pi_{H}(\alpha u)$. Moreover,

$$
\beta^{-1} \beta^{\prime}=\gamma u \gamma^{\prime-1}=b_{1} c u c^{\prime-1} b_{4}^{-1}=b_{1} b_{2} b_{4}^{-1}=b_{3},
$$

hence $d\left(\beta, \beta^{\prime}\right)=\lg \left(b_{3}\right) \leq 3$.

Lemma 5.10 Let $\alpha \in G, \beta \in \pi_{H}(\alpha)$ and $u \in \mathcal{S}$. Denote by $\theta$ the element of $T$ such that $H \theta=H \alpha$ and assume that $\theta \in M^{-1} \backslash\{1\}$. Then there exists $\beta^{\prime} \in \pi_{H}(\alpha u)$ such that $d\left(\beta, \beta^{\prime}\right) \leq 3$.

Proof Let $\theta=c \Delta^{-t}$ be the right $\Delta$-form of $\theta$ and $\ell=\lg (\theta)$. By Theorem 3.3, $\ell=\lg (H \alpha)$ and $\theta \in \operatorname{Min}(\alpha)$. Since $\theta \in M^{-1}$ and $\theta \neq 1$, by Proposition 2.3 (2), $t \geq \lg (c)$ and Corollary $2.4, \ell=t \geq \lg (c)$ and $\ell \geq 1$. Moreover, since $\theta \in T$, the element $c$ is $N$-reduced and $\omega \not_{L} c$. By Lemma 5.8, there exists $\gamma \in \operatorname{Min}(\alpha)$ such that $\beta=\alpha \gamma^{-1}$. Let $\beta_{1} \in H$ such that $\beta_{1} \theta=\gamma$. We show that $\beta_{1}=b_{1} \in N$. Suppose instead that $\beta_{1} \notin N$. Then $\beta_{1}$ is written $\beta_{1}=b_{1} \delta^{-k}$ where $k \geq 1$ and $\delta \not_{L} b_{1}$. By Lemma 3.9, the right $\Delta$-form of $\gamma$ is $\gamma=b_{1} \omega_{1} \omega_{2} \cdots \omega_{k} \Phi^{-k}(c) \Delta^{-k-\ell}$, where $\omega_{i}=\Phi^{-i+1}(\omega)$. By Corollary 2.4 and Lemma 5.7 it follows that

$$
\ell=\lg (\gamma)=\max \left(\lg \left(b_{1} \omega_{1} \omega_{2} \cdots \omega_{k} \Phi^{-k}(c)\right), k+\ell\right) \geq k+\ell>\ell:
$$


contradiction. So, $\beta_{1}=b_{1} \in N$ and $\gamma=\left(b_{1} c\right) \Delta^{-\ell}$. By Lemma 3.8, $b_{1} c$ is unmovable, hence $\gamma=\left(b_{1} c\right) \Delta^{-\ell}$ is the right $\Delta$-form of $\gamma$ and, by Corollary 2.4, $\ell=\lg (\gamma)=\max \left(\lg \left(b_{1} c\right), \ell\right)$. Thus, $\lg (c) \leq \lg \left(b_{1} c\right) \leq \ell$.

We have $\theta u=c \Delta^{-\ell} u=c u_{1} \Delta^{-\ell}$, where $u_{1}=\Phi^{-\ell}(u) \in \mathcal{S}$. Let $b_{2}=\tau\left(c u_{1}\right) \in N$ and let $c_{1} \in M$ such that $c u_{1}=b_{2} c_{1}$. We write $c_{1}$ in the form $c_{1}=\omega_{1} \omega_{2} \cdots \omega_{p} c_{2}$, where $\omega_{i}=\Phi^{-i+1}(\omega)$ for all $i \in\{1, \ldots, p\}$, and $\Phi^{-p}(\omega) \mathbb{L}_{L} c_{2}$. We show that $p \leq 1$. Assume instead that $p \geq 2$. Let $u_{1}^{\prime} \in \operatorname{Div}(\Delta)$ such that $u_{1}^{\prime} u_{1}=\Delta$. Recall that $\varphi: H \rightarrow H$ denotes the conjugation by $\delta$ in $H$. Then

$$
\omega_{1} \omega_{2} \leq_{L} c_{1} \Rightarrow b_{2} \omega_{1} \omega_{2} \leq_{L} b_{2} c_{1}=c u_{1} \Rightarrow \delta^{2} b_{2} \omega_{1} \omega_{2}=\varphi^{2}\left(b_{2}\right) \Delta^{2} \leq_{L} \delta^{2} c u_{1} .
$$

Observe that for $x \in M$ one has that $\Delta^{i} \leq_{L} x \Delta^{i}$ and $\Delta^{i} \leq_{R} \Delta^{i} x$. We have that

$$
\begin{gathered}
\omega_{1} \omega_{2} \leq_{L} c_{1} \Rightarrow \Delta^{2} \leq_{L} \delta^{2} c u_{1} \Rightarrow \Delta^{2} \leq_{R} \delta^{2} c u_{1} \Rightarrow \Delta u_{1}^{\prime} \leq_{R} \delta^{2} c \Rightarrow \Delta \leq_{R} \delta^{2} c \Rightarrow \\
\Delta \leq_{L} \delta^{2} c \Rightarrow \omega \leq_{L} \delta^{2} c \Rightarrow \omega \wedge_{L} \delta^{2}=\delta^{2} \omega \leq_{L} \delta^{2} c \Rightarrow \omega \leq_{L} c:
\end{gathered}
$$

contradiction. So, $p \leq 1$.

Let $b_{1}^{\prime}=\omega_{p}^{-1} \cdots \omega_{1}^{-1} b_{1} \omega_{1} \cdots \omega_{p} \in \Phi^{-p}(N)$ and $b_{2}^{\prime}=\omega_{p}^{-1} \cdots \omega_{1}^{-1} b_{2} \omega_{1} \cdots \omega_{p} \in$ $\Phi^{-p}(N)$. Then $b_{1} c u_{1}=b_{1} b_{2} c_{1}=b_{1} b_{2} \omega_{1} \cdots \omega_{p} c_{2}=\omega_{1} \cdots \omega_{p} b_{1}^{\prime} b_{2}^{\prime} c_{2}$. We show that there exist $b_{3}^{\prime}, b_{4}^{\prime} \in \Phi^{-p}(N)$ such that $b_{3}^{\prime} b_{4}^{\prime}=b_{1}^{\prime} b_{2}^{\prime}, \lg \left(b_{4}^{\prime} c_{2}\right) \leq \max \left(\ell-p, \lg \left(c_{2}\right)\right)$ and $\lg \left(b_{3}^{\prime}\right) \leq 2$. Set $n=\lg \left(b_{1}^{\prime} b_{2}^{\prime} c_{2}\right)$ and $m=\max \left(\ell-p, \lg \left(c_{2}\right)\right)$. We have

$$
\lg \left(c_{2}\right) \leq \lg \left(b_{2} \omega_{1} \cdots \omega_{p} c_{2}\right)=\lg \left(c u_{1}\right) \leq \lg (c)+1 \leq \ell+1 \leq \ell-p+2,
$$

hence $\ell-p \leq m \leq \ell-p+2$. So,

$$
\begin{gathered}
n-m \leq n-\ell+p \leq n-\ell+1 \leq \lg \left(\omega_{1} \cdots \omega_{p} b_{1}^{\prime} b_{2}^{\prime} c_{2}\right)-\ell+1 \\
=\lg \left(b_{1} b_{2} \omega_{1} \cdots \omega_{p} c_{2}\right)-\ell+1=\lg \left(b_{1} c u_{1}\right)-\ell+1 \leq \lg \left(b_{1} c\right)-\ell+2 \leq 2 .
\end{gathered}
$$

Let $b_{1}^{\prime} b_{2}^{\prime} c_{2}=v_{n} \cdots v_{2} v_{1}$ be the right greedy normal form of $b_{1}^{\prime} b_{2}^{\prime} c_{2}$. Suppose first that $m \geq n$. Set $b_{3}^{\prime}=1$ and $b_{4}^{\prime}=b_{1}^{\prime} b_{2}^{\prime}$. Then $\lg \left(b_{4}^{\prime} c_{2}\right)=\lg \left(b_{1}^{\prime} b_{2}^{\prime} c_{2}\right)=n \leq$ $m=\max \left(\ell-p, \lg \left(c_{2}\right)\right)$ and $\lg \left(b_{3}^{\prime}\right)=0 \leq 2$. Suppose now that $m<n$. Let $d=v_{m} \cdots v_{2} v_{1}$. Since $\lg \left(c_{2}\right) \leq m$, by Lemma 5.6 we have that $c_{2} \leq_{R} d$. Let $b_{4}^{\prime} \in M$ such that $b_{4}^{\prime} c_{2}=d$, and let $b_{3}^{\prime}=v_{n} \cdots v_{m+1}$. Then $\lg \left(b_{3}^{\prime}\right) \leq n-m \leq 2$, $\lg \left(b_{4}^{\prime} c_{2}\right) \leq m=\max \left(\ell-p, \lg \left(c_{2}\right)\right), b_{3}^{\prime} b_{4}^{\prime}=b_{1}^{\prime} b_{2}^{\prime}$, since $b_{3}^{\prime} b_{4}^{\prime} c_{2}=b_{1}^{\prime} b_{2}^{\prime} c_{2}$, and $b_{3}^{\prime}, b_{4}^{\prime} \in \Phi^{-p}(N)$, since $b_{3}^{\prime} b_{4}^{\prime}=b_{1}^{\prime} b_{2}^{\prime} \in \Phi^{-p}(N)$ and $\Phi^{-p}(N)$ is a parabolic submonoid.

Let $c^{\prime}=\Phi^{p}\left(c_{2}\right)$ and $\theta^{\prime}=c^{\prime} \Delta^{-\ell+p}$. We have $\Phi^{-p}(\omega) \mathbb{L}_{L} c_{2}$, hence $\omega \mathbb{L}_{L} c^{\prime}$. Since $\omega \leq_{L} \Delta$, this also implies that $\Delta \mathbb{L}_{L} c^{\prime}$, that is, $c^{\prime}$ is unmovable. Let $b^{\prime} \in \Phi^{-p}(N)$. Set $b=\omega_{1} \cdots \omega_{p} b^{\prime} \omega_{p}^{-1} \cdots \omega_{1}^{-1} \in N$. If $b^{\prime} \leq_{L} c_{2}$, then $\omega_{1} \cdots \omega_{p} b^{\prime}=b \omega_{1} \cdots \omega_{p} \leq_{L}$ $\omega_{1} \cdots \omega_{p} c_{2}=c_{1}$, hence $b \leq_{L} c_{1}$. Since $c_{1}$ is $N$-reduced, it follows that $b=1$, hence 
$b^{\prime}=1$. This shows that $c_{2}$ is $\Phi^{-p}(N)$-reduced, hence $c^{\prime}$ is $N$-reduced. Finally, since $\ell \geq 1$ and $p \leq 1$, we have $\ell-p \geq 0$. So, $\theta^{\prime} \in T$. On the other hand,

$$
\begin{gathered}
\alpha u=\beta \gamma u=\beta b_{1} c \Delta^{-\ell} u=\beta b_{1} c u_{1} \Delta^{-\ell}=\beta b_{1} b_{2} \omega_{1} \cdots \omega_{p} c_{2} \Delta^{-\ell} \\
=\beta b_{1} b_{2} \delta^{-p} \Delta^{p} c_{2} \Delta^{-\ell}=\beta b_{1} b_{2} \delta^{-p} c^{\prime} \Delta^{-\ell+p}=\beta b_{1} b_{2} \delta^{-p} \theta^{\prime},
\end{gathered}
$$

hence $\theta^{\prime}$ is the element of $T$ which lies in $H \alpha u$.

Set $b_{3}=\Phi^{p}\left(b_{3}^{\prime}\right)$ and $b_{4}=\Phi^{p}\left(b_{4}^{\prime}\right)$. Then $b_{3}, b_{4} \in N, \lg \left(b_{3}\right) \leq 2$, and $\lg \left(b_{4} c^{\prime}\right)=$ $\lg \left(b_{4}^{\prime} c_{2}\right) \leq \max \left(\ell-p, \lg \left(c_{2}\right)\right)=\max \left(\ell-p, \lg \left(c^{\prime}\right)\right)=\lg \left(\theta^{\prime}\right)$. Let $\gamma^{\prime}=\left(b_{4} c^{\prime}\right) \Delta^{-\ell+p}=$ $b_{4} \theta^{\prime}$. If we had $\Delta \leq_{L} b_{4} c^{\prime}$, then we would have $\omega \leq_{L} b_{4} c^{\prime}$, hence we would have $\omega \vee_{L} b_{4}=b_{4} \omega \leq_{L} b_{4} c^{\prime}$, and therefore $\omega \leq_{L} c^{\prime}$ : contradiction. So, $b_{4} c^{\prime}$ is unmovable, hence $\left(b_{4} c^{\prime}\right) \Delta^{-\ell+p}$ is the right $\Delta$-form of $\gamma^{\prime}$. By Corollary 2.4 and the above it follows that $\lg \left(\gamma^{\prime}\right)=\max \left(\lg \left(b_{4} c^{\prime}\right), \ell-p\right)=\max \left(\lg \left(c^{\prime}\right), \ell-p\right)=\lg \left(\theta^{\prime}\right)$, hence, since $\theta^{\prime} \in \operatorname{Min}(\alpha u)$, we have $\gamma^{\prime} \in \operatorname{Min}(\alpha u)$. Let $\beta^{\prime}=\alpha u \gamma^{\prime-1}$. We have $\beta^{\prime} \in \pi_{H}(\alpha u)$ by Lemma 5.8. Moreover,

$$
\begin{gathered}
\beta^{-1} \beta^{\prime}=\gamma u \gamma^{\prime-1}=b_{1} c \Delta^{-\ell} u \Delta^{\ell-p} c^{\prime-1} b_{4}^{-1}=b_{1} b_{2} c_{1} \Delta^{-p} c^{-1} b_{4}^{-1} \\
=b_{1} b_{2} \omega_{1} \cdots \omega_{p} c_{2} \Delta^{-p} c^{\prime-1} b_{4}^{-1}=\omega_{1} \cdots \omega_{p} b_{1}^{\prime} b_{2}^{\prime} c_{2} \Delta^{-p} c^{-1} b_{4}^{-1} \\
=\delta^{-p} \Delta^{p} b_{3}^{\prime} b_{4}^{\prime} c_{2} \Delta^{-p} c^{\prime-1} b_{4}^{-1}=\delta^{-p} b_{3} .
\end{gathered}
$$

So, $d\left(\beta, \beta^{\prime}\right)=\lg \left(\delta^{-p} b_{3}\right) \leq p+\lg \left(b_{3}\right) \leq 3$.

Lemma 5.11 Let $\alpha \in G, \beta \in \pi_{H}(\alpha)$ and $u \in \mathcal{S}$. Denote by $\theta$ the element of $T$ such that $H \theta=H \alpha$ and assume that $\theta \notin M \cup M^{-1}$. Then there exists $\beta^{\prime} \in \pi_{H}(\alpha u)$ such that $d\left(\beta, \beta^{\prime}\right) \leq 5$.

Proof Let $\theta=c \Delta^{-\ell}$ be the right $\Delta$-form of $\theta$. Since $\theta \notin M \cup M^{-1}$, by Proposition 2.3 and Corollary 2.4, we have $1 \leq \ell<\lg (c)$ and $\lg (\theta)=\lg (c)$. Furthermore, by Theorem 3.3, $\lg (H \alpha)=\lg (\theta)$ and so $\theta \in \operatorname{Min}(\alpha)$. Moreover, since $\theta \in T$, the element $c$ is $N$-reduced and $\omega \mathbb{L}_{L} c$. By Lemma 5.8 there exists $\gamma \in \operatorname{Min}(\alpha)$ such that $\beta=\alpha \gamma^{-1}$. Let $\beta_{1} \in H$ such that $\beta_{1} \theta=\gamma$. We show that $\beta_{1}=b_{1} \in N$. Suppose instead that $\beta_{1} \notin$ $N$. Then $\beta_{1}$ is written $\beta_{1}=b_{1} \delta^{-k}$ where $k \geq 1, b_{1} \in N$, and $\delta \not_{L} b_{1}$. By Lemma 3.9, the right $\Delta$-form of $\gamma$ is $\gamma=b_{1} \omega_{1} \omega_{2} \cdots \omega_{k} \Phi^{-k}(c) \Delta^{-k-\ell}$, where $\omega_{i}=\Phi^{-i+1}(\omega)$. Then, by Corollary 2.4 and Lemma 5.7, $\lg (\gamma)=\max \left(\lg \left(b_{1} \omega_{1} \cdots \omega_{k} \Phi^{-k}(c)\right), k+\ell\right) \geq$ $\max \left(\lg \left(\omega_{1} \cdots \omega_{k} \Phi^{-k}(c)\right), k+\ell\right)=\max (\lg (c)+k, k+\ell)>\lg (c)=\lg (\theta)$ : contradiction. So, $\beta_{1}=b_{1} \in N$ and $\gamma=\left(b_{1} c\right) \Delta^{-\ell}$. By Lemma 3.8, $b_{1} c$ is unmovable, hence $\gamma=\left(b_{1} c\right) \Delta^{-\ell}$ is the right $\Delta$-form of $\gamma$. By Corollary 2.4, $\lg (c)=\lg (\theta)=\lg (\gamma)=$ $\max \left(\lg \left(b_{1} c\right), \ell\right) \geq \lg \left(b_{1} c\right)$ and, by Lemma 3.5, $\lg \left(b_{1} c\right) \geq \lg (c)$, hence $\lg (c)=\lg \left(b_{1} c\right)$. 
We have $\theta u=c \Delta^{-\ell} u=c u_{1} \Delta^{-\ell}$, where $u_{1}=\Phi^{-\ell}(u) \in \mathcal{S}$. Let $b_{2}=\tau\left(c u_{1}\right) \in N$ and let $c_{1} \in M$ such that $c u_{1}=b_{2} c_{1}$. Since $c$ is $N$-reduced, we have $b_{2} \wedge_{L} c=1$, hence, by Theorem $2.1, \lg \left(b_{2}^{-1} c\right)=\lg \left(b_{2}\right)+\lg (c)$. It follows that

$$
\begin{gathered}
\lg (c)+1 \geq \lg \left(c u_{1}\right)=\lg \left(b_{2} c_{1}\right) \geq \lg \left(c_{1}\right)=\lg \left(b_{2}^{-1} c u_{1}\right) \\
\geq \lg \left(b_{2}^{-1} c\right)-1=\lg \left(b_{2}\right)+\lg (c)-1,
\end{gathered}
$$

hence $\lg \left(b_{2}\right) \leq 2$.

We write $c_{1}$ in the form $c_{1}=\omega_{1} \omega_{2} \cdots \omega_{p} c_{2}$, where $\omega_{i}=\Phi^{-i+1}(\omega)$ for all $i \in$ $\{1, \ldots, p\}$, and $\Phi^{-p}(\omega) \not_{L} c_{2}$. We show that $p \leq 1$ in the same way as in the proof of Lemma 5.10. Moreover, by the above,

$$
\lg \left(c_{2}\right) \geq \lg \left(b_{2} \omega_{1} \cdots \omega_{p} c_{2}\right)-\lg \left(b_{2}\right)-\lg \left(\omega_{1} \cdots \omega_{p}\right) \geq \lg \left(c u_{1}\right)-3 \geq \lg (c)-3 .
$$

Let $b_{1}^{\prime}=\omega_{p}^{-1} \cdots \omega_{1}^{-1} b_{1} \omega_{1} \cdots \omega_{p} \in \Phi^{-p}(N)$ and $b_{2}^{\prime}=\omega_{p}^{-1} \cdots \omega_{1}^{-1} b_{2} \omega_{1} \cdots \omega_{p} \in$ $\Phi^{-p}(N)$. Then $b_{1} c u_{1}=b_{1} b_{2} c_{1}=b_{1} b_{2} \omega_{1} \cdots \omega_{p} c_{2}=\omega_{1} \cdots \omega_{p} b_{1}^{\prime} b_{2}^{\prime} c_{2}$. We show now that there exist $b_{3}^{\prime}, b_{4}^{\prime} \in \Phi^{-p}(N)$ such that $b_{3}^{\prime} b_{4}^{\prime}=b_{1}^{\prime} b_{2}^{\prime}, \lg \left(b_{4}^{\prime} c_{2}\right)=\lg \left(c_{2}\right)$ and $\lg \left(b_{3}^{\prime}\right) \leq 4$. Set $n=\lg \left(b_{1}^{\prime} b_{2}^{\prime} c_{2}\right)$ and $m=\lg \left(c_{2}\right)$. We have $n \geq m$ by Lemma 3.5 and, by the above,

$$
\begin{gathered}
n=\lg \left(b_{1}^{\prime} b_{2}^{\prime} c_{2}\right) \leq \lg \left(\omega_{1} \cdots \omega_{p} b_{1}^{\prime} b_{2}^{\prime} c_{2}\right)=\lg \left(b_{1} c u_{1}\right) \leq \lg \left(b_{1} c\right)+1 \\
=\lg (c)+1 \leq \lg \left(c_{2}\right)+4=m+4 .
\end{gathered}
$$

Let $b_{1}^{\prime} b_{2}^{\prime} c_{2}=v_{n} \cdots v_{2} v_{1}$ be the right greedy normal form of $b_{1}^{\prime} b_{2}^{\prime} c_{2}$. Set $d=$ $v_{m} \cdots v_{2} v_{1}$. By Lemma 5.6 we have $c_{2} \leq_{R} d$. Let $b_{4}^{\prime} \in M$ such that $b_{4}^{\prime} c_{2}=d$ and let $b_{3}^{\prime}=v_{n} \cdots v_{m+1}$. We have $b_{3}^{\prime} b_{4}^{\prime} c_{2}=b_{1}^{\prime} b_{2}^{\prime} c_{2}$, hence $b_{3}^{\prime} b_{4}^{\prime}=b_{1}^{\prime} b_{2}^{\prime}$. In particular, $b_{3}^{\prime}, b_{4}^{\prime} \in \Phi^{-p}(N)$, since $b_{1}^{\prime} b_{2}^{\prime} \in \Phi^{-p}(N)$ and $\Phi^{-p}(N)$ is a parabolic submonoid. Furthermore, by the above, $\lg \left(b_{3}^{\prime}\right)=n-m \leq 4$ and $\lg \left(b_{4}^{\prime} c_{2}\right)=\lg (d)=m=\lg \left(c_{2}\right)$.

Let $c^{\prime}=\Phi^{p}\left(c_{2}\right)$ and let $\theta^{\prime}=c^{\prime} \Delta^{-\ell+p}$. We show in the same way as in the proof of Lemma 5.10 that $\theta^{\prime}=c^{\prime} \Delta^{-\ell+p}$ is the right $\Delta$-form of $\theta^{\prime}$, that $\omega \mathbb{L}_{L} c^{\prime}$, that $\theta^{\prime} \in T$, and that $\theta^{\prime} \in H \alpha u$. Set $b_{3}=\Phi^{p}\left(b_{3}^{\prime}\right)$ and $b_{4}=\Phi^{p}\left(b_{4}^{\prime}\right)$. Then $b_{3}, b_{4} \in N, \lg \left(b_{3}\right) \leq 4$, and $\lg \left(b_{4} c^{\prime}\right)=\lg \left(b_{4}^{\prime} c_{2}\right)=\lg \left(c_{2}\right)=\lg \left(c^{\prime}\right)$. Let $\gamma^{\prime}=\left(b_{4} c^{\prime}\right) \Delta^{-\ell+p}=b_{4} \theta^{\prime}$. If we had $\Delta \leq_{L} b_{4} c^{\prime}$, then we would have $\omega \leq_{L} b_{4} c^{\prime}$, hence we would have $\omega \vee_{L} b_{4}=$ $b_{4} \omega \leq_{L} b_{4} c^{\prime}$, and therefore $\omega \leq_{L} c^{\prime}$ : contradiction. So, $b_{4} c^{\prime}$ is unmovable, hence $\left(b_{4} c^{\prime}\right) \Delta^{-\ell+p}$ is the right $\Delta$-form of $\gamma^{\prime}$. By Corollary 2.4 and the above it follows that $\lg \left(\gamma^{\prime}\right)=\max \left(\lg \left(b_{4} c^{\prime}\right), \ell-p\right)=\max \left(\lg \left(c^{\prime}\right), \ell-p\right)=\lg \left(\theta^{\prime}\right)$. So, since $\theta^{\prime} \in \operatorname{Min}(\alpha u)$, we have $\gamma^{\prime} \in \operatorname{Min}(\alpha u)$. Let $\beta^{\prime}=\alpha u \gamma^{\prime-1}$. We have $\beta^{\prime} \in \pi_{H}(\alpha u)$ by Lemma 5.8. Furthermore,

$$
\begin{gathered}
\beta^{-1} \beta^{\prime}=\gamma u \gamma^{\prime-1}=b_{1} c \Delta^{-\ell} u \Delta^{\ell-p} c^{\prime-1} b_{4}^{-1}=b_{1} b_{2} \omega_{1} \cdots \omega_{p} c_{2} \Delta^{-p} c^{\prime-1} b_{4}^{-1} \\
=\omega_{1} \cdots \omega_{p} b_{1}^{\prime} b_{2}^{\prime} c_{2} \Delta^{-p} c^{-1} b_{4}^{-1}=\delta^{-p} \Delta^{p} b_{3}^{\prime} b_{4}^{\prime} c_{2} \Delta^{-p} c^{\prime-1} b_{4}^{-1}=\delta^{-p} b_{3} .
\end{gathered}
$$


Thus, $d\left(\beta, \beta^{\prime}\right)=\lg \left(\delta^{-p} b_{3}\right) \leq p+\lg \left(b_{3}\right) \leq 5$.

Proof of Theorem 5.5 Follows directly from Lemma 5.9, Lemma 5.10 and Lemma 5.11 .

\section{Acknowledgments}

The first author acknowledges partial support from the Spanish Government through grant number MTM2017-82690-P and through the "Severo Ochoa Programme for Centres of Excellence in R\&D” (SEV-2015-0554)

\section{References}

[1] Y Antolín, Counting subgraphs in fftp graphs with symmetry, preprint, arXiv:1802.05213.

[2] E Brieskorn, Die Fundamentalgruppe des Raumes der regulären Orbits einer endlichen komplexen Spiegelungsgruppe, Invent. Math. 12 (1971), 57-61.

[3] E Brieskorn, Sur les groupes de tresses [d'après V. I. Arnol'd], Séminaire Bourbaki, 24éme année (1971/1972), Exp. No. 401, pp. 21-44. Lecture Notes in Math., Vol. 317, Springer, Berlin, 1973.

[4] E Brieskorn, K Saito, Artin-Gruppen und Coxeter-Gruppen, Invent. Math. 17 (1972), 245-271.

[5] F Callegaro, D Moroni, M Salvetti, Cohomology of affine Artin groups and applications, Trans. Amer. Math. Soc. 360 (2008), no. 8, 4169-4188.

[6] R Charney, Artin groups of finite type are biautomatic, Math. Ann. 292 (1992), no. 4, 671-683.

[7] R Charney, Geodesic automation and growth functions for Artin groups of finite type, Math. Ann. 301 (1995), no. 2, 307-324.

[8] C De Concini, M Salvetti, Cohomology of Coxeter groups and Artin groups, Math. Res. Lett. 7 (2000), no. 2-3, 213-232.

[9] P Dehornoy, Groupes de Garside, Ann. Sci. École Norm. Sup. (4) 35 (2002), no. 2, 267-306.

[10] P Dehornoy, Alternating normal forms for braids and locally Garside monoids, J. Pure Appl. Algebra 212 (2008), no. 11, 2413-2439.

[11] P Dehornoy, F Digne, E Godelle, D Krammer, J Michel, Foundations of Garside theory, EMS Tracts in Mathematics, 22, European Mathematical Society (EMS), Zürich, 2015. 
[12] P Dehornoy, L Paris, Gaussian groups and Garside groups, two generalisations of Artin groups, Proc. London Math. Soc. (3) 79 (1999), no. 3, 569-604.

[13] P Deligne, Les immeubles des groupes de tresses généralisés, Invent. Math. 17 (1972), 273-302.

[14] D B A Epstein, J W Cannon, D F Holt, S V F Levy, M S Paterson, W P Thurston, Word processing in groups, Jones and Bartlett Publishers, Boston, MA, 1992.

[15] P Flajolet, R Sedgewick, Analytic combinatorics, Cambridge University Press, Cambridge, 2009.

[16] F A Garside, The braid group and other groups, Quart. J. Math. Oxford Ser. (2) 20 (1969), 235-254.

[17] E Godelle, Parabolic subgroups of Garside groups, J. Algebra 317 (2007), no. 1, 1-16.

[18] E Godelle, Parabolic subgroups of Garside groups II: ribbons, J. Pure Appl. Algebra 214 (2010), no. 11, 2044-2062.

[19] E Godelle, L Paris, $K(\pi, 1)$ and word problems for infinite type Artin-Tits groups, and applications to virtual braid groups, Math. Z. 272 (2012), no. 3-4, 1339-1364.

[20] D F Holt, Garside groups have the falsification by fellow-traveller property, Groups Geom. Dyn. 4 (2010), no. 4, 777-784.

[21] H Van der Lek, The homotopy type of complex hyperplane complements, $\mathrm{Ph}$. D. Thesis, Nijmegen, 1983.

[22] L Paris, $K(\pi, 1)$ conjecture for Artin groups, Ann. Fac. Sci. Toulouse Math. (6) 23 (2014), no. 2, 361-415.

[23] M Picantin, The conjugacy problem in small Gaussian groups, Comm. Algebra 29 (2001), no. 3, 1021-1039.

[24] J Tits, Normalisateurs de tores. I. Groupes de Coxeter étendus, J. Algebra 4 (1966), 96-116.

Departamento de Matemáticas, Facultad de Ciencias, Universidad Autónoma de Madrid, 28049 Madrid, Spain

IMB, UMR 5584, CNRS, Univ. Bourgogne Franche-Comté, 21000 Dijon, France

yago.anpi@gmail.com, lparis@u-bourgogne.fr 